\title{
The Comprehensive Assessment of Neurodegeneration and Dementia: Canadian Cohort Study
}

\author{
Howard Chertkow, Michael Borrie, Victor Whitehead, Sandra E. Black, \\ Howard H. Feldman, Serge Gauthier, David B. Hogan, Mario Masellis, \\ Katherine McGilton, Kenneth Rockwood, Mary C. Tierney, Melissa Andrew, \\ Ging-Yuek R. Hsiung, Richard Camicioli, Eric E. Smith, Jennifer Fogarty, \\ Joseph Lindsay, Sarah Best, Alan Evans, Samir Das, Zia Mohaddes, Randi Pilon, \\ Judes Poirier, Natalie A. Phillips, Elizabeth MacNamara, Roger A. Dixon, \\ Simon Duchesne, Ian MacKenzie, R. Jane Rylett
}

\begin{abstract}
Background: The Comprehensive Assessment of Neurodegeneration and Dementia (COMPASS-ND) cohort study of the Canadian Consortium on Neurodegeneration in Aging (CCNA) is a national initiative to catalyze research on dementia, set up to support the research agendas of CCNA teams. This cross-country longitudinal cohort of 2310 deeply phenotyped subjects with various forms of dementia and mild memory loss or concerns, along with cognitively intact elderly subjects, will test hypotheses generated by these teams. Methods: The COMPASS-ND protocol, initial grant proposal for funding, fifth semi-annual CCNA Progress Report submitted to the Canadian Institutes of Health Research December 2017, and other documents supplemented by modifications made and lessons learned after implementation were used by the authors to create the description of the study provided here. Results: The CCNA COMPASS-ND cohort includes participants from across Canada with various cognitive conditions associated with or at risk of neurodegenerative diseases. They will undergo a wide range of experimental, clinical, imaging, and genetic investigation to specifically address the causes, diagnosis, treatment, and prevention of these conditions in the aging population. Data derived from clinical and cognitive assessments, biospecimens, brain imaging, genetics, and brain donations will be used to test hypotheses generated by CCNA research teams and other Canadian researchers. The study is the most comprehensive and ambitious Canadian study of dementia. Initial data posting occurred in 2018, with the full cohort to be accrued by 2020. Conclusion: Availability of data from the COMPASS-ND study will provide a major stimulus for dementia research in Canada in the coming years.
\end{abstract}

RÉSUMÉ : Évaluation complète d'une étude de cohorte canadienne portant sur la démence et la neuro-dégénérescence. Contexte : L'évaluation globale de la neuro-dégénérescence et de la démence (COMPASS-ND), étude de cohorte du Consortium canadien en neuro-dégénérescence associée au vieillissement $(\mathrm{CCNV})$, représente une initiative nationale visant à promouvoir la recherche portant sur la démence et à soutenir les programmes de recherche des équipes du CCNV. Totalisant 2310 sujets recrutés partout au pays, cette cohorte longitudinale regroupe des individus fortement « phénotypés » qui présentent diverses formes de démence et de pertes de mémoire légères. En plus de sujets âgés dont les fonctions cognitives sont intactes, ces 2310 sujets ont permis de valider les hypothèses formulées par les équipes du CCNV. Méthodes : Nous avons utilisé de nombreux documents pour décrire cette étude : le protocole de la COMPASS-ND ; la demande initiale de subvention ; le cinquième rapport d'étape semi-annuel du CCNV soumis aux Instituts de recherche

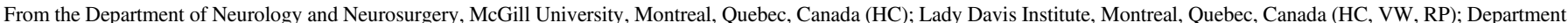

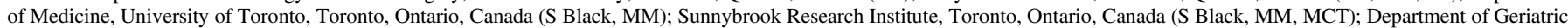

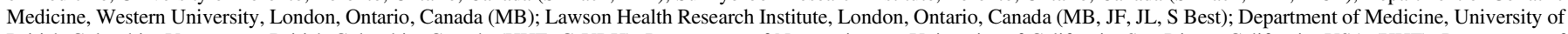

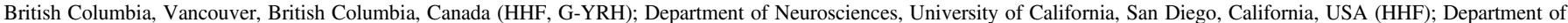

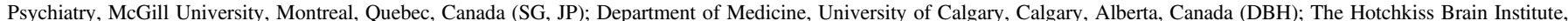

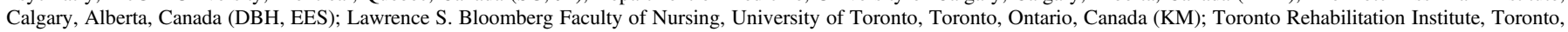

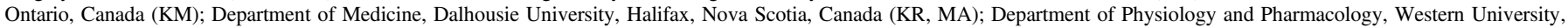

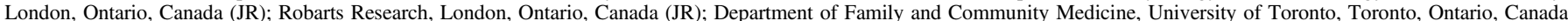

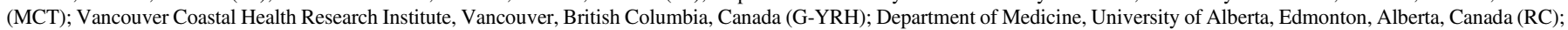

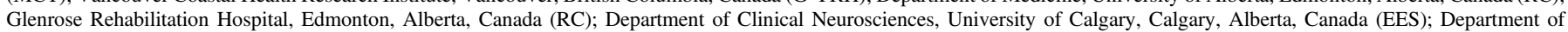

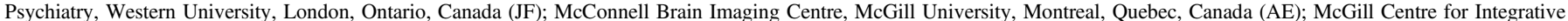

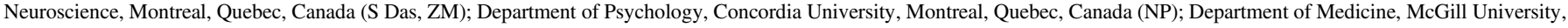

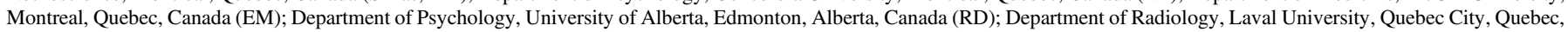

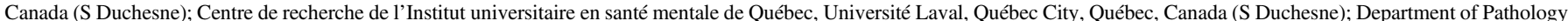

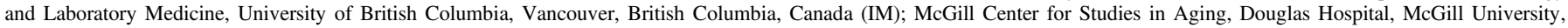
Montreal, Quebec, Canada (SG).

Received October 16, 2018. Final Revisions Submitted February 22, 2019. Date of Acceptance February 25, 2019

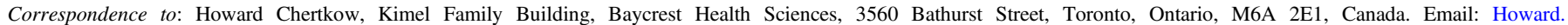
chertkow@mcgill.ca 
en santé du Canada (IRSC) en décembre 2017 ; ainsi que d'autres documents produits à la suite de modifications consécutives à la mise en œuvre de ce projet. Résultats : L'étude de cohorte COMPASS-ND du CCNV inclut des participants de partout au Canada dont les divers états cognitifs sont associés à des maladies neurodégénératives ou au risque d'en souffrir. Ils feront l'objet d'un large éventail d'examens expérimentaux, cliniques, génétiques et d'imagerie afin d'aborder de manière spécifique les causes, le diagnostic, le traitement et la prévention de ces états cognitifs chez les personnes âgées. Les données obtenues à la suite d'évaluations cliniques et cognitives, ainsi que celles issues d'échantillons biologiques, d'imagerie cérébrale, de tests génétiques et de dons de cerveaux, seront utilisées pour tester les hypothèses générées par les équipes de recherche du CCNV et d'autres chercheurs canadiens. Cette étude constitue donc à ce jour l'étude canadienne la plus complète et la plus ambitieuse au sujet de la démence. La présentation des données initiales ayant eu lieu en 2018, la cohorte devrait atteindre sa taille maximale d'ici à 2020. Conclusion : La disponibilité des données de l'étude COMPASS-ND stimulera considérablement la recherche sur la démence au Canada au cours des prochaines années.

Keywords: Cohort study, Dementia, Frontotemporal dementia, Biomarkers, Diagnosis, Alzheimers

doi:10.1017/cjn.2019.27

Can J Neurol Sci. 2019; 46: 499-511

\section{BACKGROUND}

The Canadian Institutes of Health Research (CIHR) established the Canadian Consortium on Neurodegeneration in Aging (CCNA) as the major national vehicle to coordinate and strengthen Canadian research on Alzheimer's Disease (AD) and other neurodegenerative diseases (NDDs). CCNA was intended to foster innovative and collaborative research, and brings together most of the established senior Canadian researchers in dementia. Twenty teams were established with expertise spanning from basic science to clinical areas of research. Details of CCNA are available at www.ccna-ccnv.ca.

It quickly became evident that numerous clinical questions remain to be answered - for instance, what are the clinical and biomarker profiles of mixed dementia subjects compared to other forms of dementia? Fifteen of the twenty CCNA teams wished to test hypotheses and obtain data involving human subjects with various forms of memory complaints or loss and NDDs. The decision was made to create a single national study within CCNA that would include cohorts of individuals with various cognitive concerns and/or NDDs. Participants would be recruited from memory, stroke, and Parkinson's disease and movement disorders clinics. This would allow the gathering of complementary data from across the country. The cohort study, a Comprehensive Assessment of Neurodegeneration and Dementia (COMPASS-ND) creates the infrastructure nationally for recruiting centers and data collection, with most sites based in academic clinical research centers in Canada. Thirty-one data collection sites across the country have been established for recruitment. Many of the principal investigators also serve within the Canadian Consortium of Centres for Clinical Cognitive Research (C5R), a national clinical trials network. Over 60 researchers working in a number of committees spent 2 years designing the final protocol that will gather the information required for answering the 106 core research questions posed by the 15 CCNA teams.

The study will ultimately enroll 1650 individuals with different NDDs and/or degrees of memory loss or concern, as well as a comparison group of cognitively intact older subjects $(n=660)$ being collected through separate financial support obtained from the CIHR Big Data in Dementia grant, the Broad and deep Analysis in Neurodegeneration (BRAIN) study. The enrolment of COMPASS-ND is currently (May 2019) at 50\% and should be completed by 2020 . In this paper, we describe the methodology and components of the COMPASS-ND study.

\section{Methods}

Members of the Research Executive Committee (REC) as well as the COMPASS-ND Protocol Implementation Team collaborated in the preparation of this manuscript. The submitted and approved protocols for the COMPASS-ND study were reviewed. The initial grant proposal and the fifth semi-annual progress report, which was submitted to CIHR in December 2017, were utilized. News and press items as well as policies and operating guidelines posted on the website (www.ccna-ccnv.ca) were also reviewed and incorporated into the Results section. Finally, modifications made after implementation of the initial protocol are noted where relevant.

\section{Results}

\section{Conceptual Framework}

COMPASS-ND was designed to recruit participants with a range of the NDDs of aging. The NDDs encountered in older persons consist of a family of related clinical-pathological entities. AD is the most common form of NDD and contributes in whole or in part to approximately two-thirds of dementia cases in older adults. ${ }^{1}$ Vascular dementia accounts for up to $20 \%$ of dementia cases, second only to $\mathrm{AD} .^{2}$ While vascular cognitive impairment is not infrequently the result of a stroke, most commonly, it is not preceded by a discrete cerebrovascular event. ${ }^{3}$ Mixed dementia - cognitive impairment where multiple brain pathologies are present - represents the most prevalent type of cognitive impairment. ${ }^{4}$ Frontotemporal dementia (FTD) accounts for $20 \%$ of early onset (i.e., $<65$ years of age) dementia cases with symptoms beginning on average around 45-65 years of age. ${ }^{5}$ Symptoms of FTD progress at a rapid, steady rate with survival after diagnosis between 2 and 10 years in most. ${ }^{6}$ Dementia with Lewy Bodies accounts for about $5 \%$ of cases of dementia. $^{7}$

To date, a collaborative approach to research that spans a wide breadth of presumably related NDD has rarely been undertaken. COMPASS-ND was designed to include and span a wide range of conditions. This enables comparisons to be made across 
disease states. This also enables the identification of common and unique factors across these diseases. Furthermore, creation of core assessment platforms that include multiple modalities will give us a more comprehensive evaluation of brain health in each of these disease states than is currently available. Using these multiple modalities will provide the opportunity to identify clinical, imaging, genetic, and other biological markers associated with the different neurodegenerative and vascular pathologies in the pathogenesis of adult onset dementia.

There is an urgency to be able to identify those who will develop NDD before their functional abilities are compromised, so that treatment may be enacted to maintain independence. It is well established that those with mild cognitive impairment (MCI) are at increased risk for progression to a dementia, ${ }^{8}$ but it remains a subject for debate what proportion of patients with MCI will eventually develop dementia and who they are. ${ }^{9,10}$ In recent years, there has been increased interest in those who have a complaint about their cognition but no objective impairment. This population has been referred as having subjective cognitive impairment (SCI). There is evidence that they are at increased risk of developing dementia. ${ }^{11,12}$ Because of this interest in early diagnosis and developing predictor biomarkers, it was elected to enroll MCI (in its broadest terms) and SCI cohorts.

The primary goal of COMPASS-ND is to create a panCanadian cohort that will, for the first time, integrate a wide range of experimental, clinical, cognitive, imaging, and genetic information and be a valuable resource for researchers. Using current diagnostic criteria (see online Supplementary material), our goal was to establish research cohorts that are well characterized in terms of their demographics, pre-morbid history, cognitive function, genetic profile, neural structure and pathology, and important biomarkers and biological indices. These data will then be made available to the research teams, allowing them access to a more extensive dataset than is typically possible.

\section{General Study Objectives}

For each of the study cohorts, we are:

1. Collecting data on sociodemographic characteristics, medical and social history, physical examination, and past and current medications, as well as gait assessment and sensory function (audition and vision), and questions related to cognitive reserve and reproductive history.

2. Collecting neuropsychological data assessing episodic, associative and prospective memory, attention, language, visuoperceptual abilities, processing speed, and executive function.

3. Carrying out collection of biosamples of blood, saliva, and urine in all participants, and cerebrospinal fluid (CSF) collection in at least $25 \%$ of the cohort.

4. Collecting MRI data according to Canadian Dementia Imaging Protocol (CDIP).

5. Identifying psychosocial issues to inform the development of programmes to support patients and families.

\section{Study Design}

This is a longitudinal observational cohort study that allows embedded interventions for subgroups. The cohorts are defined by clinical cognitive syndromes with agreed on diagnostic criteria.

\section{Table 1: COMPASS-ND enrolment goals}

\begin{tabular}{l|c}
\hline Diagnosis & Minimum number \\
\hline Cognitively intact elderly & 660 \\
\hline SCI/SCD & 300 \\
\hline MCI without vascular lesions & 400 \\
\hline Subcortical ischemic V-MCI & 200 \\
\hline Mild AD & 150 \\
\hline Mild dementia of mixed etiology & 200 \\
\hline Lewy body disease/Parkinson's dementia & 200 \\
\hline FTD & 200 \\
\hline TOTAL & 2310 \\
\hline
\end{tabular}

A total of 1650 memory-impaired/concerned subjects, including those with SCI/Subjective Cognitive Decline (SCD), vascular MCI (V-MCI)/MCI, AD/Mixed dementia, Lewy Body Dementia (LBD)/Parkinson's disease dementia (PDD)/Parkinson's disease MCI (PD-MCI), and FTD/primary progressive aphasias (PPA), along with 660 cognitively normal individuals will be enrolled into this study from 31 centers (i.e., memory clinics affiliated with the C5R, select Canadian stroke clinics, and select Canadian movement disorders clinics). Patients will be 50-90 years of age and recruited to ensure adequate representation of male and female participants in each diagnostic subgroup. We will ensure that the male/female distribution within each diagnostic group mirrors that found in the general population. The assessment was initially planned as a cross-sectional study, but with additional funding from the BRAIN grant, there will be a planned follow-up evaluation at 2-year-post-recruitment. The goal is to repeat the entire set of procedures below (except genetic testing) for this second assessment. If CCNA goes on to a Phase II renewal, further longitudinal follow-ups will be arranged.

Study participants involved in the study will undergo comprehensive baseline evaluations, including clinical and neuropsychological assessment, biospecimen collection, genomics, and MRI neuroimaging. Participants may still continue to be a part of the study if they decline to undertake certain procedures. The minimum level of participation required for study inclusion is the MRI scan and clinical assessment. The collected data will be cleaned and made available to CCNA investigators as well as others eventually through the Longitudinal Online Research and Imaging System (LORIS) database. ${ }^{13}$

A decision was made not to conduct a strict "natural history" study. We felt that this deeply phenotyped cohort of participants could be targeted for small scale 6-24-month intervention studies if willing. We have chosen to carry out primarily non-pharmacological intervention studies of diet, exercise, and cognitive stimulation. Specific CCNA teams are conducting these intervention studies using COMPASS-ND subjects with MCI and SCI/SCD. The initial COMPASS-ND assessment will function as a "pre-intervention" evaluation, providing considerable savings in terms of cost and participant assessment burden.

The protocol was designed to harmonize, where possible, with ongoing Canadian and international studies of aging and dementia, in particular, the Canadian Longitudinal Study of Aging 
Time window for completion: 90 days

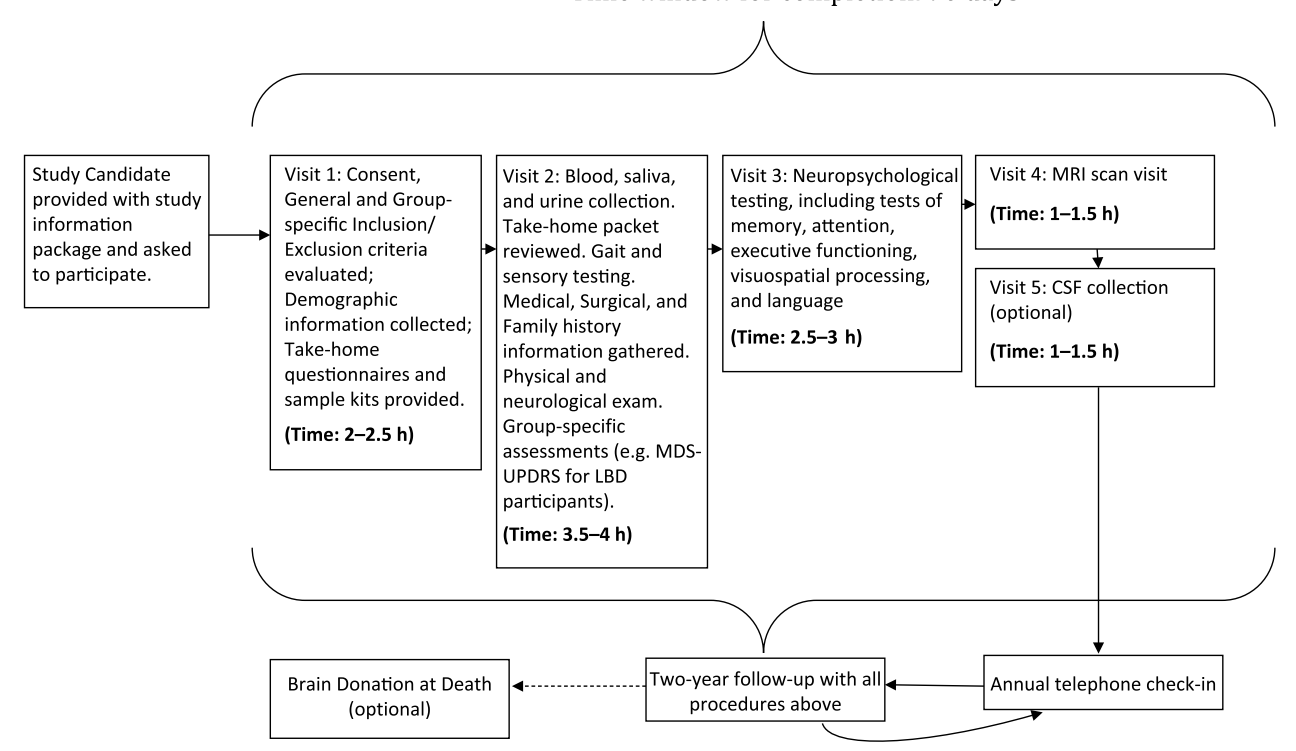

Figure 1: Flow plan of the assessments in COMPASS-ND.

(CLSA), ${ }^{14}$ The Ontario Neurodegenerative Disease Research Initiative (ONDRI), Le Consortium pour l'identification précoce de la maladie d'Alzheimer - Québec (CIMA-Q; Consortium for the Early Identification of AD - Québec), and the Alzheimer's Disease Neuroimaging Initiative (ADNI) ${ }^{15}$ to allow for crossstudy comparisons. For instance, the diet researchers designed the dietary questionnaire to be administered, ${ }^{16}$ and it is also being administered as part of the CLSA. One of the CCNA teams is focused on gait and cognition, and designed similar gait measures to gather necessary data for COMPASS-ND as well as an associated cohort in Ontario. ${ }^{17}$

\section{Participants}

Leaving aside the cohort of cognitively intact older persons, a total of 1650 subjects (see Table 1) are to be enrolled. Sample size was determined for adequate testing of various planned hypotheses, and so that sex distribution of the groups would be adequate to enable the analysis of how sex impacts the expression of different types of dementia. A group of cognitively intact older subjects $(n=660)$ will be recruited for normative data especially on the experimental tasks that have been developed for this study. A subset of this group $(n=200)$ will undergo the full COMPASSND protocol while the remainder will participate in a shortened protocol that excludes an MRI and biosample collection.

Basic decisions around the COMPASS-ND cohort arose after discussions with the International Scientific Advisory Board (ISAB) of the CCNA. An important one was to focus on mixed pathology and mixed dementias. It is anticipated that most recruited dementia cases will have mixed pathology, co-morbidities, and evidence of vascular disease. Exclusionary criteria are being kept to a minimum. This was motivated by three considerations: recent work has demonstrated extensive mixed pathology in most older persons with dementia, ${ }^{18}$ and we wished to enroll a more ecologically accurate dementia cohort; while we recognized that other studies have brought forth a wealth of information on highly selected "ready for drug trial" cases of AD and MCI, the majority of those seen in the clinical practice are excluded from these studies and are generally under-studied; and research on the vascular aspects of the dementias is particularly strong in Canada, ${ }^{19-21}$ and we felt important contributions could be made from less restricted recruitment.

The specific inclusion and exclusion criteria for enrolling subjects in the study are described below.

\section{General Inclusion Criteria (All Conditions)}

Subjects must meet each of the following criteria for enrolment into the study:

1. Written informed consent must be obtained and documented.

2. Sufficient proficiency in English or French to undergo assessment. Good proficiency on the Language Experience and Proficiency Questionnaire (LEAP-Q), ${ }^{22}$ a self-assessment measure of linguistic ability for both speaking and understanding of English or French, is required for inclusion into the study.

3. Geographic accessibility (living within $1 \mathrm{~h}$ to the study site) is required.

4. Must have a study partner who sees the participant weekly, and who can participate as required in the protocol.

\section{General Exclusion Criteria (All Conditions)}

1. The presence of other significant known chronic brain disease (e.g., moderate to severe chronic static leukoencephalopathy including previous traumatic injury), multiple sclerosis, a serious developmental handicap, malignant tumors, Huntington's disease, and other rarer brain illnesses

2. Ongoing alcohol or drug abuse

3. Subject does not have a study partner

4. Individuals where English or French is not sufficiently proficient

5. Total score on the Montreal Cognitive Assessment $(\mathrm{MoCA})<13$ 
6. Symptomatic stroke within the previous year

7. Unwilling or unable to undergo MRI scan

\section{Assessments}

The five assessment visits include extensive clinical testing and questionnaires; auditory, visual, and gait measures; an in-depth neuropsychological battery; physical examination; 3T MRI scan; and blood, saliva, fecal, and urine collection for multiple biosample measures. If the subject agrees, a lumbar puncture is carried out at Visit 5 . There will be a 12 -week window from time of consent to complete all screening and baseline procedures. The overall assessment plan is seen in Figure 1.

(A) (Visit 1) Consent, Demographics, and Screening Clinical Assessments (Estimated Time: 2-2.5 h)

The following study procedures are performed:

- Written informed consent prior to study procedures

- Assessment of inclusion/exclusion criteria

- Hearing and vision assessment

- Sociodemographic, physical activity, and nutrition and oral health information

- Logical Memory 1 and 2 from Wechsler Memory ScaleRevised $^{23}$

- Clinical Dementia Rating scale ${ }^{24}$

- Consortium to Establish a Registry for Alzheimer's Disease (CERAD) Word List Recall ${ }^{25}$

- $\mathrm{MoCA}^{26}$ (those with SCI/SCD must have a score $\geq 25$; all participants must have a score $\geq 13$ )

- Lawton Brody Instrumental Activities of Daily Living scale ${ }^{27}$

- Benson Figure Recall ${ }^{28}$

- Mini-mental state examination (MMSE) ${ }^{29}$

- National Alzheimer's Coordinating Center (NACC) Uniform Data Set frontotemporal lobar dementia (FTLD) Module to delineate clinical features of PPA and behavioral variant FTD (bvFTD) ${ }^{30}$

- Geriatric Depression scale and Generalized Anxiety Disorder 7-item Scale

\section{(B) Self-Administered Questionnaires to be Completed between Visits 1 and 2}

A take home package is filled out by caregivers at home including information on:

- Hobbies and leisure activities, tobacco and alcohol consumption, activities of daily living, quality of life, end of life care, social network, support, and activities

- Formal questionnaires on: adverse childhood experiences (optional), the Neuropsychiatric Inventory, ${ }^{31}$ the Parkinson's Disease Questionnaire (PDQ-39), ${ }^{32}$ and the UPDRS version revised by the Movement Disorders Society MDSUPDRS, parts IB - II (LBD, PDD, PD-MCI, PD-NCI, and FTD spectrum only) $)^{33}$

- FTD spectrum subjects' caregivers will also fill out scales on the Behavioral Inhibition Scale Interpersonal Reactivity Self-Monitoring, along with an Apathy Inventory

In addition, there will be an at-home fecal collection for microbiome assessment.
(C) Visit 2: Completion of Clinical and Physical Assessments (Estimated Time: 2.5-3 h)

At visit 2, the following information is gathered and study procedures performed by the study nurse:

- Physical measurements, health perception, fatigue, falls history and balance assessment, walking speed at 4 and 6 $\mathrm{m}$, and grip strength

- Formal vision assessment, olfaction assessment, and hearing assessment

- Reported sleep features, caregiver burden and cognitive fluctuations

- Clinical details of disease onset and progression, medical, mental health, surgical history, and family history

- Physical and neurological examination

- Hachinski Ischemic Scale ${ }^{34}$

- Clinical diagnosis reassessment (Diagnostic criteria are attached as online Supplemental material)

- Collection of fasting blood, saliva, urine, and buccal swab

(D) Visit 3: Neuropsychological Assessment (Estimated Time: 3-3.5 h)

At visit 3, the following information will be gathered and study procedures performed (also see Table 2):

- Visuoperceptual and construction ability

- Learning and memory

- Attention, working memory, and processing speed

- Executive function

- Speech and language

\section{(E) Visit 4: MRI Scan (Estimated Time: 1 h)}

At visit 4, a magnetic resonance imaging scan is performed on a $3 \mathrm{~T}$ magnet machine. It may be done prior to the clinical and neuropsychological assessments but must be done prior to the lumbar puncture.

\section{(F) Visit 5: Lumbar Puncture (Estimated Time: 1 h)}

At visit 5, a lumbar puncture is performed optionally if consented to by the participant.

\section{(G) Follow-Up}

All cohort subjects and/or informants will be contacted via telephone on an annual basis to ensure continued willingness to participate and request permission to consult their treating physician to determine if there has been any diagnostic change. If there is consent for a brain donation upon the participant's death, their brain will be collected according to established protocols. All subjects will be seen in person 2 years after recruitment and undergo the same procedures as in visits $1-4$.

\section{Clinical evaluation}

The clinical assessment consists of demographics, past and current medical/psychiatric conditions, past and current medications, reproductive history, childhood adversity, past and current sleep quality/patterns, past and current pain status, nutrition, 


\section{Table 2: Neuropsychological battery}

\begin{tabular}{|c|c|c|c|}
\hline Test battery & & & \\
\hline Domain & Test & Description & Time (min) \\
\hline Premorbid IQ & WAIS-III Vocabulary* & Expressive vocabulary & 10 \\
\hline \multirow[t]{3}{*}{ Visuoperceptual and construction ability } & Object Decision Test -BORB & Object perception & 5 \\
\hline & Judgment of Line Orientation (split half) & Visual perception & 10 \\
\hline & BVMT Copy & Visuoconstruction & 3 \\
\hline \multirow[t]{5}{*}{ Learning and memory } & RAVLT $^{*}$ & Word-list learning & $5-8$ \\
\hline & BVMT $^{*}$ & Figure learning & $10-15$ \\
\hline & CCNA/CIMA-Q Face-Name Matching & Associative recall & $10-15$ \\
\hline & Digit Symbol - Incidental Recall & Incidental associative recall & 5 \\
\hline & Envelope Test* & Prospective memory & 5 \\
\hline \multirow{3}{*}{$\begin{array}{l}\text { Attention and working memory and } \\
\text { processing speed }\end{array}$} & WAIS-III Digit Span forward and backward & Attention span and working memory & $5-10$ \\
\hline & WAIS-III Digit Symbol-Coding & Psychomotor processing speed & 5 \\
\hline & CCNA Simple and Choice Reaction Time & Reaction time & 10 \\
\hline \multirow[t]{6}{*}{ Executive function } & D-KEFS Phonemic Fluency* & Phonemic (letter) fluency & 5 \\
\hline & Reitan Trail Making Test (A \& B)* & Attention switching & $5-10$ \\
\hline & D-KEFS Color Word Interference & Interference resolution; switching & $7-12$ \\
\hline & $\begin{array}{l}\text { CCNA/CIMA-Q Sentence Completion } \\
\text { Test* }\end{array}$ & Inhibition (verbal) & 10 \\
\hline & NACC Social Norms Questionnaire & Knowledge of appropriate social mores & 5 \\
\hline & NACC Social Behavior Observer Checklist & Social behavior and self-awareness & 5 \\
\hline \multirow[t]{9}{*}{ Speech and language } & D-KEFS Semantic Fluency* & Semantic (category) fluency & 3 \\
\hline & Cookie Theft Picture Description & Free speech & 2 \\
\hline & NACC Word Reading Test & Reading of regular and irregular words & $2-3$ \\
\hline & $\begin{array}{l}\text { NACC Semantic Word-Picture Matching } \\
\text { Test }\end{array}$ & Word recognition and comprehension & 5 \\
\hline & NACC Semantic Associates Test & Semantic memory & 5 \\
\hline & NACC Northwestern Anagram Test & Grammatical knowledge & 10 \\
\hline & NACC Sentence Repetition Test & Oral repetition & 1 \\
\hline & NACC Noun and Verb Naming Subtests & Naming of objects and actions & 6 \\
\hline & NACC Sentence Reading Test & Reading & 1 \\
\hline
\end{tabular}

$\mathrm{BORB}=$ Birmingham Object Recognition Battery; BVMT = Brief Visuospatial Memory Test; D-KEFS = Delis-Kaplan Executive Function System; NACC $=$ National Alzheimer's Coordinating Center; RAVLT $=$ Rey Auditory Verbal Learning Test; WAIS = Weschler Adult Intelligence Scale .

*Tests monitored $100 \%$ of the time. All testing sessions are audio-recorded.

lifestyle factors including physical and social activity, and other domains of interest. The clinical items were chosen to overlap as much as possible with several large-scale provincial initiatives (i.e., CIMA-Q ${ }^{35}$ ONDRI $^{36}$ ).

\section{Neuropsychological Evaluation}

The neuropsychology test battery was designed to assess a comprehensive range of cognitive domains and be sensitive to a range of ability levels, ranging from older adults with relatively intact cognitive abilities to participants with diagnosed dementia. This cognitive test battery is independent from the cognitive screening tests used to classify participants above (Section 5A, Screening Visit). Neuropsychological testing will provide a characterization of the participant's cognitive profile to discriminate dementia types and track changes with dementia progression.
Domains assessed will be broad based and include attention, learning and memory, speech production, language, executive function, and visuospatial function (see Table 2 below). The Movement Disorder Society Task Force Guidelines were used to design the battery of clinical tests for Parkinson's disease patient cohort. The test battery was chosen to overlap as much as possible with several large-scale provincial initiatives (i.e., CIMA-Q ${ }^{35}$ $\mathrm{ONDRI}^{36}$ ) and several provincial disease-specific studies (FTD in British Columbia ${ }^{37}$ and Parkinson's disease in Alberta ${ }^{38}$ ). The battery requires between 2 and $3 \mathrm{~h}$ to administer.

\section{Biosamples}

Dementia biomarkers may be measured in blood, ${ }^{39} \mathrm{CSF},{ }^{40}$ and saliva. ${ }^{41}$ They can help us to better understand the mechanisms and effects of neurodegeneration and provide potential 
Table 3: List of blood and CSF biomarkers in planned analysis

\begin{tabular}{|c|c|}
\hline \multicolumn{2}{|l|}{ Blood } \\
\hline Electrolytes ( $\mathrm{Na}, \mathrm{K}, \mathrm{Cl}$, and bicarbonate) & Androstenedione \\
\hline Creatinine & Luteinizing hormone \\
\hline Urea & Follicle-stimulating hormone \\
\hline Insulin level & Dehydroepiandrosterone sulfate \\
\hline Glucose & Estrone sulphate \\
\hline $\begin{array}{l}\text { Liver function (AST, ALT, ALP, and } \\
\text { bilirubin) }\end{array}$ & Testosterone (total) \\
\hline B12 & Dihydrotestosterone \\
\hline Homocysteine & Estrone \\
\hline C-reactive protein & Estradiol \\
\hline Insulin-like growth factor 1 & Prolactin \\
\hline Tumor necrosis factor-alpha & Cortisol \\
\hline Interleukin 6 & Sex hormone-binding globulin \\
\hline Total cholesterol & Thyroid stimulating hormone \\
\hline Triglycerides & Calcium \\
\hline High density lipoproteins & Albumin \\
\hline Low density lipoproteins & 25-OH vitamin D level \\
\hline ApoA-1 & Ferritin \\
\hline ApoB & Cystatin c \\
\hline Alpha-1-antitrypsin & $\begin{array}{l}\text { Brain derived neurotrophic factor (serum } \\
\text { and RNA) }\end{array}$ \\
\hline Biliverdin & $\begin{array}{l}\text { Vascular endothelial growth factor (serum } \\
\text { and RNA) }\end{array}$ \\
\hline \multicolumn{2}{|l|}{ Adrenocorticotropic hormone } \\
\hline \multicolumn{2}{|c|}{ CSF } \\
\hline \multicolumn{2}{|l|}{ Amyloid-beta $1-42$} \\
\hline \multicolumn{2}{|l|}{ Total tau } \\
\hline \multicolumn{2}{|l|}{ Phosphorylated tau } \\
\hline Alpha synuclein & \\
\hline
\end{tabular}

diagnostic markers to aid in earlier diagnosis of these diseases. Blood, urine, and saliva will be collected on all participants, who will also be asked if they are willing to undergo a lumbar puncture to provide CSF samples (based on our review of similar observational studies that included the option of a lumbar puncture, this is a conservative estimate). ${ }^{42}$

A total of $53.4 \mathrm{~mL}$ of blood will be collected via venipuncture in 10 tubes. A total of $4 \mathrm{~mL}$ of saliva will be collected by passive drool in one tube. A total of $40 \mathrm{~mL}$ of CSF will be collected via lumbar puncture. The samples will be aliquoted into $500-\mu \mathrm{L} \mathrm{V}$ bottom, screw-top tubes (Matrix Tubes, Thermo Fisher Scientific, Carlsbad, CA) and stored in a $-80^{\circ} \mathrm{C}$ fridge until they can be transported via cryoshipper to the Canadian Biosample Repository (CBSR) in Edmonton for more permanent storage.

Core biomarkers will be assessed at the clinical laboratory of the Jewish General Hospital in Montreal (Dr. E. MacNamara, director). The samples are being collected, handled, stored, and shipped according to rigorously established standard operating procedures.
A set of core established biomarkers will be assessed for all participants including general measures of health, measures of sex-related hormones, inflammation, lipid metabolism, and oxidative stress. Further experimental measures will be assessed on sub-samples of participants based on the needs of the researchers. See Table 3 and Figures 2 and 3 for the Blood and Fluids Processing Plan.

\section{MRI Scanning}

A workshop of Canadian imaging experts led by Dr. Simon Duchesne developed the CDIP, which will be used in COMPASS-ND. ${ }^{43}$ The particular sequences used are all standardized across MRI machines throughout the country and include 3D T1, PD/T2, FLAIR, gradient echo, resting state fMRI, and Diffusion Tensor Imaging (DTI). The 3 tesla (3T) MRI scans planned as part of COMPASS-ND will allow comparison with ADNI data. CDIP will also be used in a growing number of other Canadian brain study cohorts, including ONDRI, CIMA-Q, and the Canadian Alliance for Healthy Hearts and Minds. ${ }^{44}$ By using exactly the same sequences in these different studies, the potential to combine and compare across them becomes possible.

The following analyses of MRI images will be carried out on each subject when possible:

1. Core Tissue Classification and Volumetrics. Structural MRI will be performed using acquisition protocols that have already been standardized across vendor platforms and are compatible with the current ADNI protocols, the VCI Harmonization criteria, and guidelines for detecting amyloid-related imaging abnormalities. The core protocol allows tissue compartment classification, including a sophisticated subtype analysis of vascular lesions, classified as lacunes, and as lesions in the periventricular or deep nuclei/white matter (not connected to the ventricles). This pipeline has been developed over the last decade and combines automatic and user-assisted manual steps.

2. Automated Hippocampal Segmentation. This will be contracted out to "True Positive," a spin-off McGill company that has developed real-time volumetrics for clinical trials. The data will be loaded into LORIS.

3. DTI. Preprocessing will initially be based on standard approaches using FMRIB Software Library that have proven effective in measuring significantly preserved white matter integrity in elderly bilinguals versus monolinguals (mean age $=70.5$ years., $\mathrm{SD}=3$ years.) based on significant differences in fractional anisotropy and radial diffusivity.

4. Resting State fMRI. Preprocessed resting-state data sets will be provided within CCNA for each subject utilizing automated pipelines.

5. Visual Review for Vascular Lesions. This will be carried out according to the Standards for Reporting Vascular Changes on Neuroimaging (Strive) protocol. ${ }^{45}$ Visual classification of infarcts, lacunes, white matter hyperintensity burden by the Fazekas scale, ${ }^{46}$ microbleeds, and superficial siderosis will be carried out by MRI Core Labs at the University of Calgary and Sunnybrook Hospital (University of Toronto) by qualified MR readers. Infarcts will be classified by size, location, and vascular territory, 


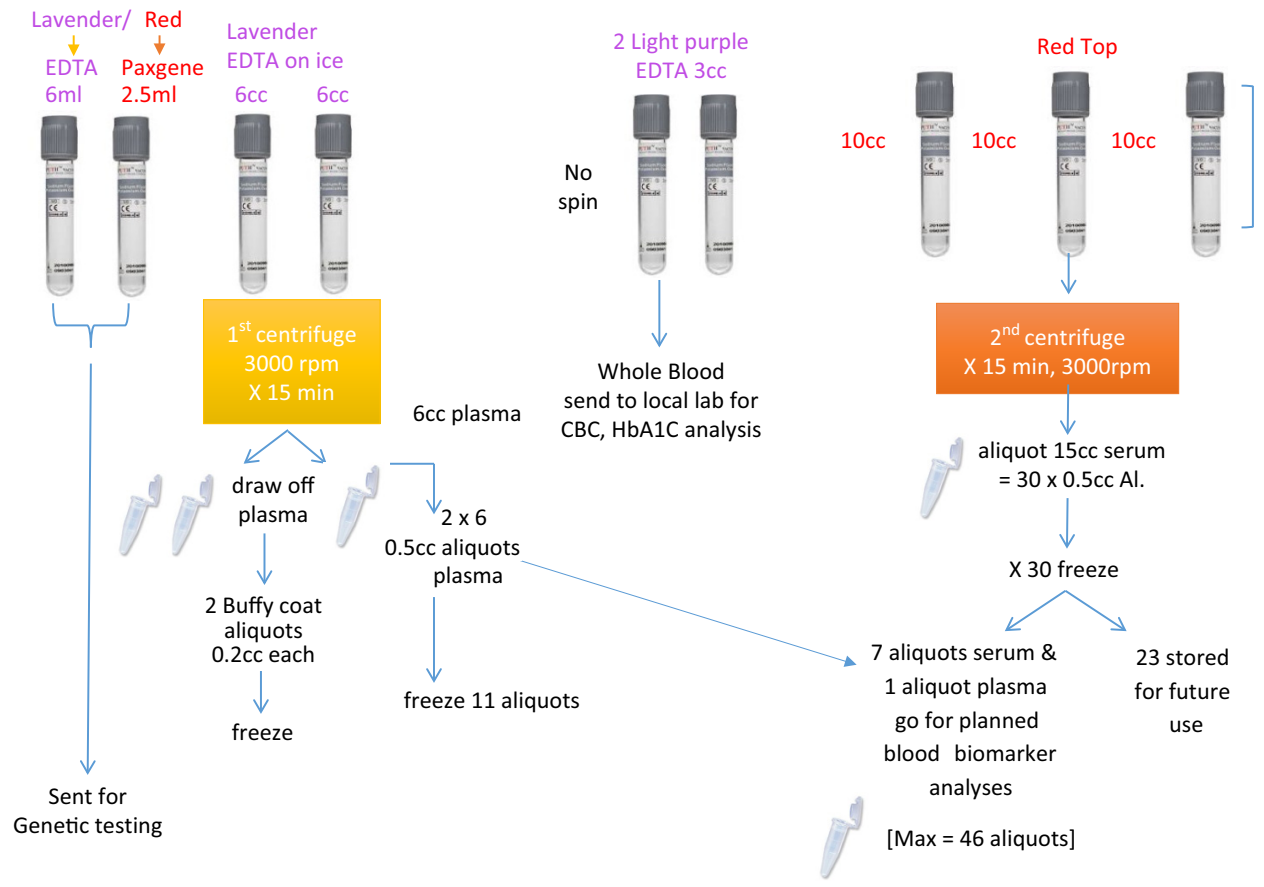

Figure 2: Blood sample processing.

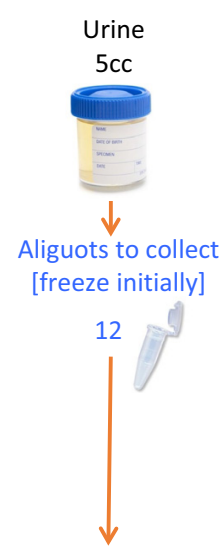

Store for future use

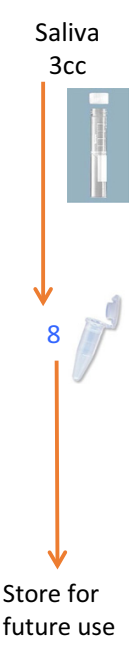

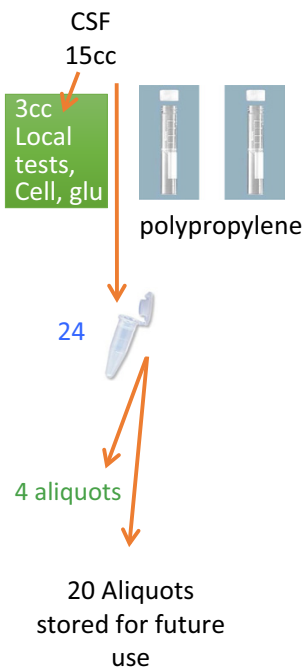

use
Fecal and buccal swab

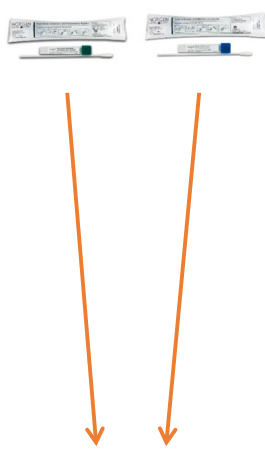

Sent for

microbiome analysis

Figure 3: Processing of CSF and other biofluids.

while microbleeds will be classified by location and by pattern according to Boston criteria for cerebral amyloid angiopathy.

\section{Genetics}

We will carry out extensive genotyping of all subjects within COMPASS-ND. ${ }^{47}$ All samples will be genotyped at the Mt. Sinai Clinical Genomics Centre (CGC)/University of Toronto using the Affymetrix UK Biobank Axiom array chip suitable for genetic association analysis for all NDDs. This chip will provide a costeffective common, cross-disease platform for the discovery of: (a) all known mutations $(\sim 1000)$ in genes known to cause $\mathrm{AD}$; (b) 20,000 key tagging SNPs for all genome-wide significant loci published in human GWAS for PD, FTLD, ALS, AD, and stroke together with the top $\sim 1000$ SNPs just below genomewide significance; (c) $\sim 349,000$ coding sequence variants with a frequency of $>1 \%$ in the human genome. It will provide 820,967 markers overall. All data will be archived in LORIS to allow us to evaluate the common and rare genetic susceptibility components in our subjects, giving a deep genotypic phenotyping to match the depth of study of clinical and imaging data. Uniquely, we will be able for the first time to test for rare monogenic and complex polygenic susceptibility across the full range of patients with neurodegenerative disorders. Familial cases with high opportunity for discovery of novel genes and novel variants will undergo whole-exome sequencing. 


\section{Microbiome Assessment}

Emergent research is linking the microbiome to a myriad of clinical conditions, including inflammation, several gastrointestinal disorders, asthma, obesity, Type 2 diabetes, as well as brain disorders such as anxiety, depression, autism, and stress response. ${ }^{48,49}$ Factors released by the gut microbiome have been shown recently to directly modify function of microglia, the brain's immune cells, providing a mechanistic link between the periphery and brain inflammation. Gut microbiota makes or affects several neurotransmitters, and sectioning the vagus nerve decreases the occurrence of Parkinson's disease. ${ }^{49,50}$ This suggests that any large data set acquisition should include a microbiome analysis. Although there are several "smoking guns" that the microbiota may impact on Alzheimer's, ${ }^{49,51-53}$ there have not been defined cohort studies to directly examine this. There is considerable expertise in Canada under Dr. Brett Finlay and his University of British Columbia team to carry out these analyses.

We are collecting fecal and oral samples for bacterial DNA studies from our COMPASS-ND cohort. ${ }^{53}$ A family member at home collects the fecal samples after the first visit. The oral scraping is taken at Visit 2. Both collections will use the Norgen Biotek Swab Collection and Preservation System. All samples are then shipped to the CBSR in Edmonton and from there to the laboratory of Dr. Brett Finlay at UBC for microbiome processing.

This cutting edge assessment will give an insight into the inflammatory state of NDD subjects, which will complement the measures of inflammation in blood, and CSF being collected. This initiative would directly address this, with the added benefit of having all the accompanying clinical data, making it an extremely novel and important aspect to further understanding AD. ${ }^{54}$

\section{Brain Donation and Brain Banking}

For many NDDs, a definitive diagnosis can only be made through analysis of brain tissue generally acquired at autopsy. Tissue pathology is still the "gold standard" for diagnosis and is an important tool to examine epigenetic and proteomic aspects of NDDs. ${ }^{55,56}$ COMPASS-ND will work closely with the Canadian ADNI Brain Donation and Neuropathology Network (CABIN) led by Dr. Ian Mackenzie. Funds from CABIN will collect ADNI brains and COMPASS-ND brains. We are setting up a national brain donation system, standardized dementia neuropathology protocols, and creating a set of national brain tissue banks for dementia studies. This will be a national resource that will allow correlations between pathology and longitudinal clinical, neuropsychological, and imaging data. Assessment of brain tissue postmortem will provide definitive diagnoses for the various types of NDDs that enrolled in COMPASS-ND. Results derived from the anonymized brain tissue samples will be attached to the other data related to that COMPASS-ND subject. This will allow clinical pathological-genetic-imaging correlations to be studied. Brain tissue from well-characterized cases will be made available for Canadian researchers.

\section{Data Storage and Management}

COMPASS-ND data being collected on participants are being uploaded and stored in the LORIS database. The LORIS data management system is based at McGill University and is directed by Dr. Alan Evans. ${ }^{13}$ It is a modular and extensible web-based data management system that integrates all aspects of a multicenter study: from heterogeneous data acquisition (imaging, clinical, behavior, and genetics) to storage, processing, and ultimately dissemination. It is storing data that have been processed to remove any direct identifiers of an individual study participant. Study subjects are being assigned a unique anonymized study identification number that will be used to store their data. LORIS CCNA data will be accessible to all CCNA members, and ultimately to the entire Canadian and international research community through the GAAIN Portal system. ${ }^{57}$ The subjects' confidentiality will be maintained. Information about study subjects will be made publicly available to the extent permitted by the applicable laws and regulations. In CCNA publications, only group data will be reported.

\section{Partnerships}

It should be noted that while the questionnaires, examinations, psychometric testing, and biomarkers were planned to answer specific hypotheses, we also took into consideration a need to synergize with other partners in Canada who were to be potential sources of subjects. A partnership has been established with an overlapping natural history cohort in Ontario (ONDRI) and a cohort in Quebec (CIMA-Q). Many of the data collection sites for COMPASS-ND are also involved in those studies. It is hoped that several hundred of their research subjects may become subsequent subjects in COMPASS-ND. To this end, about one-third of the data to be collected was designed to overlap in a complementary fashion with their procedures and questions, with the goal of avoiding unnecessary subject burden and cost. Contracts and data sharing arrangements have been undertaken. Further overlap in psychometric testing was geared toward comparisons with the CLSA ${ }^{14}$ which is a population-based study of aging in over 50,000 individuals who will be followed for 20 years. The study includes a psychometric evaluation as well as biospecimen collection but currently no brain imaging.

\section{Normative Comparison Group}

The original plan of COMPASS-ND did not include funding for a separate cognitively intact elderly control group. Additional funding through the BRAIN grant will allow us to establish a control group of cognitively intact older individuals as previously described. These individuals are being accrued under direction of CCNA team leaders Drs. Natalie Philips and Roger Dixon. In addition to providing the necessary control data for comparisons with the patient participant groups, the COMPASS-ND will provide normative neuropsychological data on the COMPASSND cognitive battery to be used by memory clinics across Canada.

\section{Intervention Studies within COMPASS-ND}

We are leveraging the considerable opportunity offered by the deep phenotyping within COMPASS-ND to launch three interventional studies with largely non-pharmacological approaches. The economy of this approach is that the subject is assessed initially for entry into COMPASS-ND, then undergoes the intervention, and then is restudied and reassessed. The initial COMPASS-ND assessment thus becomes the baseline for the study, with considerable saving of costs. The downside of this 
approach is that these subjects (particularly if they are in the active intervention arm and particularly if the intervention proves successful) are then lost as part of a "natural history of progression" study. It is our view, however, that the benefits of developing new interventions for the NDDs outweigh this loss and is the most appropriate approach to follow.

The intervention studies consist of the SYNERGIC (Synchronizing Exercise Remedies in Gait and Cognition) study, a 6-month study of intensive exercise plus or without Vitamin $\mathrm{D}$, carried out in MCI individuals $(n=160)$; the ENGAGE study (Exploring Novel Group Activities for Geriatric Enrichment) - a 6-month study of cognitive stimulation interventions (or placebo) carried out by Team 10 under Drs. Sylvie Belleville and Nicole Anderson in 200 SCI individuals in Toronto and Montreal; and the LEAD study (Lifestyle Exercise and Dementia), a 1-year study of dietary and exercise interventions carried out in MCI and SCI individuals.

\section{Administration and Governance of COMPASS-ND}

The CCNA is administered as a grant from CIHR. The REC directs the scientific management of CCNA. On the REC, two members (Dr. Howard H. Feldman and Dr. Michael Borrie) represent the COMPASS-ND study. The REC has bi-weekly teleconferences and has several in-person meetings a year. As head of the Clinical Platform of CCNA, Dr. Michael Borrie plays a critical role in the implementation of COMPASS-ND and is supported by Sarah Best, Dr. Jennifer Fogarty, Cynthia Di Prospero, and Nimi Bassi. The Protocol Implementation Team of COMPASS-ND meets every week to deal with day-to-day operational issues. The reimbursement of site costs and payment for participant accrual are managed by the central administration of CCNA.

\section{Data Access and Publication}

The guiding principle of COMPASS-ND is to maximize access to and use of the valuable data being collected, and to catalyze the Canadian dementia research community. The clinical, neuropsychological, imaging, biomarker, and genomic data from each clinical site or laboratory within CCNA will be managed and uploaded centrally to the LORIS database. A Publications and Data Access Committee (PDAC) was established as a sub-committee of the CCNA REC. PDAC policy does not in itself restrict analysis of CCNA data, which might be with a view to publication, pilot testing, or education. Access to and analyses of COMPASS-ND study data by CCNA investigators will be granted automatically upon request of access. However, as part of their team and group agendas, many CCNA investigators (the teams, platforms, and REC) have already submitted a list of "Protected planned projects and publications," which constitutes a writing plan for CCNA for the first 12 months after the full COMPASS-ND cross-sectional data have been collected, uploaded into LORIS, and locked. This 12-month period will allow the designated teams to write the planned papers. All CCNA investigators are expected to honor the priority of the list of "Protected" topics, since the teams and platforms that designed the COMPASS-ND study did so as a means to address specific hypotheses. After the quarantine period, any CCNA investigator can undertake/publish research on any question that has not been addressed yet. To do so, the CCNA investigator must submit a summary of the project/publication proposed to the PDAC. The PDAC will verify that the project has not already been submitted by a CCNA team of platform. After the quarantine period, non-CCNA investigators may be granted access to CCNA acquired data upon submission of a project outline supporting their data access request. Ultimately, all COMPASS-ND data will become part of the GAAIN portal open access system.

A Biological Samples Access Committee was also set up to establish policy and procedures for the disposition and allocation of the biological samples that are obtained from participants in CCNA studies. It will also review applications from CCNA and non-CCNA researchers for studies that propose to use CCNA biological specimens, with specified criteria. An accounting of the biological specimens available to the research community through CCNA will be maintained in the CCNA

\section{The Ethical and Legal Aspects of COMPASS-ND}

Paramount in COMPASS-ND is subject confidentiality. We have worked closely with the CCNA Ethical Legal and Social Implications of the research (ELSI) Committee under the direction of Dr. Serge Gauthier to deal with ethical issues raised by such a multi-centre study. COMPASS-ND will be conducted in compliance with the protocol, International Conference on Harmonization Good Clinical Practice and the applicable regulatory requirements. Any medically significant incidental findings (as judged by the site research physician) will be communicated to the patient's personal physician, who will decide on appropriate subsequent communication of the finding to the participant along with possible treatment options. COMPASS-ND also provides an opportunity to assess to what degree knowledge of biomarkers (CSF, imaging, and blood and genetics) would alter the diagnosis and management of physicians. A CCNA Clinical Diagnosis Committee is currently exploring how to carry out ethical relaying of biomarker information back to treating physicians in order to track the impact of this information on diagnosis and treatment.

\section{Involvement in International Collaborations}

COMPASS-ND will be an important achievement for the Canadian dementia research community. However, it is clear that our cohorts do not supply sufficient sample sizes to answer many questions about gene/environment interactions. We will partner with international groups to pool deeply phenotyped subjects. Internationally, similar (and similarly sized) cohorts are being collected in other countries. A Memorandum of Understanding with the UK Dementia Platform has already been brought into effect, and other international agreements are being pursued. It is hoped that CCNA will also be positioned to negotiate Canada's participation in international dementia prevention studies in the future.

\section{Conclusion}

In the COMPASS-ND study, a national dementia cohort of 2310 "deeply phenotyped" affected individuals, and cognitively intact controls, is being established that will catalyze research across both basic and clinical research fields in dementia in Canada. This will be unique in focusing on disease heterogeneity, mixed dementias, and comparing and contrasting different dementia types in great detail. 
Novel intervention studies of diet, exercise, and cognitive stimulation have been leveraged off of these deeply characterized COMPASS-ND subjects with mild memory loss, and these are ongoing. Possible new therapeutic approaches may result. Moving forward, we anticipate further developing initiative that can prevent progression from MCI to dementia and ensure the relevance of dementia research to people living now at high risk of developing dementia within a short period.

We believe COMPASS-ND will allow CCNA to lead the way in bringing cooperation and collegiality to dementia research and researchers. COMPASS-ND represents a uniquely Canadian partnership with scientists and clinicians working together and pooling resources to obtain information out of the reach of individual researchers. Working together, we can make even faster progress toward our common goal of dealing with the challenge of NDDs.

\section{ACKNOWLEDGEMENTS}

We thank Nathalie Bélanger, Pascale Léon, and Shelley Solomon for help in the preparation of this manuscript.

\section{Funding}

The CCNA is supported by an infrastructure and operating grant from the CIHR (Grant no. CNA-137794) and the following partners:

Alberta Prion Research Institute

Alzheimer's Research UK

Alzheimer Society of Canada

Canadian Nurses Foundation

Fonds de recherche du Québec - Santé

Michael Smith Foundation for Health Research

New Brunswick Health Research Foundation

Nova Scotia Health Research Foundation

Ontario Brain Institute

Pfizer Inc.

Robin and Barry Picov Family Foundation

Sanofi

Saskatchewan Health Research Foundation

Women's Brain Health Initiative

The writing of this paper was also supported in part by a foundation grant from CIHR awarded to HC. None of the funders had any role in the preparation of this manuscript.

\section{DISCLOSURES}

Dr. HC reports grants from CIHR, other from Alberta Prion Research Institute, other from Alzheimer's Research UK, other from Alzheimer Society of Canada, other from Canadian Nurses Foundation, non-financial support from Fonds de recherche du Québec - Santé, other from Michael Smith Foundation for Health Research, other from New Brunswick Health Research Foundation, other from Nova Scotia Health Research Foundation, nonfinancial support from Ontario Brain Institute, non-financial support from Pfizer Inc., other from Robin and Barry Picov Family Foundation, other from Sanofi, other from Saskatchewan Health Research Foundation, and other from Women's Brain Health Initiative, during the conduct of the study. Ms. S Best, Mr. SD, Dr. JF, Dr. JR, Mr. JL, Mr. ZM, Ms. RP, and Mr. VW have nothing to disclose. Drs. RC, RD, AE, SG, DH, IM, EM, $\mathrm{KM}$, NP, JP, KR, and MCT report grants from CIHR, during the conduct of the study. Dr. MA reports grants from CHIR, during the conduct of the study; reports grants from GSK, grants from Pfizer, and grants from Sanofi, outside the submitted work. Dr. SEB reports funding to the institution from CIHR for the Compass-ND study, and personal fees from Novartis, Merck, Pfizer, Eli Lilly, Biogen/Medscape, and grants to the institution from Novartis, Eli Lilly, GE Healthcare, Biogen Idec, Genentech, Optina, outside of submitted work. Dr. MB reports grants from CIHR, during the conduct of the study; grants from Ontario Brain Institute/ONDRI study, outside the submitted work. Dr. S Duchesne reports grants from CHIR, during the conduct of the study; reports other from True Positive Medical Devices Inc., from Montreal, Canada, outside the submitted work. Dr. HF reports grants from CIHR, Brain Canada, National Institutes of Health: Alzheimer Disease Cooperative Study U19AG10483-26, Biohaven Pharmaceuticals, Toyama Pharmaceuticals, and development grant funding from Probiodrug, during the conduct of the study; service agreements with Eisai Pharmaceuticals, Genentech/ Roche Pha, maceuticals, Banner Health Institute, Samus Therapeutics, Merck Pharmaceuticals, Tau RX, Arkuda Therapeutics, and Samumed; speaker fees from World Events Forum, Medscape, Optum, and San Diego Academy of Family Physicians; travel expenses from Axon Neurosciences, Alion Pharmaceuticals, Probiodrug, and Dominantly Inherited Alzheimer's Disease. Dr. G-YR Hsiung reports grants from CHIR, during the conduct of the study; reports grants from CIHR and NIH, professorship support from Alzheimer Society of BC, clinical trials investigator support from AstraZeneca, Eli Lilly, and Roche/Genentech, outside the submitted work. Dr. MM reports grants from CIHR, during the conduct of the study; other from Associate Editor, Current Pharmacogenomics and Personalized Medicine, personal fees from Bioscape Medical Imaging CRO, personal fees from GE Healthcare, personal fees from UCB, grants from CIHR, grants from Early Researcher Award, Ministry of Economic Development and Innovation of Ontario, grants from Ontario Brain Institute, grants from Sunnybrook AFP Innovation Fund, grants from Alzheimer's Drug Discovery Foundation (ADDF), grants from Brain Canada, grants from Heart and Stroke Foundation Centre for Stroke Recovery, grants from Weston Brain Institute, personal fees from Novartis, personal fees from Henry Stewart Talks, other from Novartis, grants from Roche, grants from Washington University, grants from Teva, and other from Teva, outside the submitted work. Dr. EES reports grants from CIHR, during the conduct of the study; and grants from CIHR and grants from Brain Canada, outside the submitted work. Dr. KR reports being founder and president of DGI Clinical, which during the initial study period had contracts on individualized outcome assessment with Roche, Otsuka, and Lundbeck.

\section{Statement of Authorship}

$\mathrm{HC}$ conceived and oversaw the writing; MB and VW contributed to the writing; S Black, HF, SG, DH, MM, KM, KR, MT, MA, GRH, RC, ES, JF, JL, S Best, AE, S Das, ZM, RP, JP, NP, EM, RD, S Duchesne, JR, and IM each reviewed, critiqued, and edited the manuscript. 


\section{ETHICS APPROVAL AND CONSENT}

The Jewish General Research Ethics Board approved the COMPASS-ND study.

\section{SupPlementary Material}

To view supplementary material for this article, please visit https://doi.org/10.1017/cjn.2019.27.

\section{REFERENCES}

1. Alzheimer Society of Canada. Rising tide: the impact of dementia on Canadian society. Toronto: Alzheimer Society of Canada; 2010.

2. Gorelick PB, Scuteri A, Black SE, et al. Vascular contributions to cognitive impairment and dementia: a statement for healthcare professionals from the American Heart Association/American Stroke Association. Stroke. 2011;42:2672-713.

3. Iadecola C. The pathobiology of vascular dementia. Neuron. 2013;80(4):844-66.

4. Schneider JA, Boyle PA, Arvanitakis Z, Bienias JL, Bennett DA. Subcortical infarcts, Alzheimer's disease pathology, and memory function in older persons. Ann Neurol. 2007;62(1):59-66.

5. Snowden JS, Neary D, Mann DM. Frontotemporal dementia. Br J Psychiatry. 2002;180:140-3.

6. Kertesz A. Frontotemporal dementia/Pick's disease. Arch Neurol. 2004;61(6):969-71.

7. Hogan DB, Fiest KM, Roberts JI, et al. The prevalence and incidence of dementia with Lewy bodies: a systematic review. Can J Neurol Sci. 2016;43(Suppl 1):S83-95.

8. Petersen RC, Negash S. Mild cognitive impairment: an overview. CNS Spectr. 2008;13(1):45-53

9. Morris JC, Mild cognitive impairment is early-stage Alzheimer disease. Arch Neurol. 2006;63:15-16.

10. Sachdev PS, Lipnicki DM, Crawford J, et al. Factors predicting reversion from mild cognitive impairment to normal cognitive functioning: a population-based study. PLoS One. 2013;8(3): e59649.

11. Reisberg B, Gauthier S. Current evidence for subjective cognitive impairment (SCI) as the pre-mild cognitive impairment (MCI) stage of subsequently manifest Alzheimer's disease. Int Psychogeriatr. 2008;20(1):1-16.

12. Jessen $\mathrm{F}$, Wolfsgruber $\mathrm{S}$, Wiese $\mathrm{B}$, et al. AD dementia risk in late $\mathrm{MCI}$, in early MCI, and in subjective memory impairment. Alzheimers Dement. 2014;10(1):76-83.

13. Das S, Zijdenbos AP, Harlap J, Vins D, Evans AC. LORIS: a webbased data management system for multi-center studies. Front Neuroinform. 2011;5:37.

14. Raina PS, Wolfson C, Kirkland SA, et al. The Canadian longitudinal study on aging (CLSA). Can J Aging. 2009;28(3):221-9.

15. Weiner MW, Veitch DP, Aisen PS, et al. Impact of the Alzheimer's disease neuroimaging initiative, 2004 to 2014. Alzheimers Dement. 2015;11(7):865-84.

16. Shatenstein B, Nadon S, Godin C, Ferland G. Development and validation of a food frequency questionnaire. Can J Diet Pract Res. 2005;66(2):67-75.

17. Montero-Odasso M, Pieruccini-Faria F, Bartha R, et al. Motor phenotype in neurodegenerative disorders: gait and balance platform study design protocol for the Ontario Neurodegenerative Research Initiative (ONDRI). J Alzheimers Dis. 2017;59(2): 707-21.

18. Schneider JA, Arvanitakis Z, Leurgans SE, Bennett DA. The neuropathology of probable Alzheimer disease and mild cognitive impairment. Ann Neurol. 2009;66(2):200-8.

19. Iturria-Medina Y, Hachinski V, Evans AC. The vascular facet of late-onset Alzheimer's disease: an essential factor in a complex multifactorial disorder. Curr Opin Neurol. 2017;30(6): 623-9.

20. Smith EE, Beaudin AE. New insights into cerebral small vessel disease and vascular cognitive impairment from MRI. Curr Opin Neurol. 2018;31(1):36-43.
21. Nestor SM, Misic B, Ramirez J, et al. Small vessel disease is linked to disrupted structural network covariance in Alzheimer's disease. Alzheimers Dement. 2017;13(7):749-60.

22. Marian V, Blumenfeld HK, Kaushanskaya M, The Language Experience and Proficiency Questionnaire (LEAP-Q): assessing language profiles in bilinguals and multilinguals. J Speech Lang Hear Res. 2007;50(4):940-67.

23. Wechsler D. Wechsler memory scale - revised. San Antonio: The Psychological Corporation; 1987.

24. Morris JC. The Clinical Dementia Rating (CDR): current version and scoring rules. Neurology. 1993;43(11):2412-4.

25. Welsh KA, Butters N, Mohs RC, et al. The Consortium to Establish a Registry for Alzheimer's Disease (CERAD). Part V. A normative study of the neuropsychological battery. Neurology. 1994;44(4):609-14.

26. Nasreddine ZS, Phillips NA, Bedirian V, et al. The Montreal Cognitive Assessment, MoCA: a brief screening tool for mild cognitive impairment. J Am Geriatr Soc. 2005;53(4):695-9.

27. Lawton M, Brody EM. Assessment of older people: self-maintaining and instrumental activities of daily living. Gerontologist. 1969;9:179-196.

28. Possin KL, Laluz VR, Alcantar OZ, Miller BL, Kramer JH. Distinct neuroanatomical substrates and cognitive mechanisms of figure copy performance in Alzheimer's disease and behavioral variant frontotemporal dementia. Neuropsychologia. 2011; 49(1):43-8.

29. Folstein MF, Folstein SE, McHugh PR. Mini-mental state: a practical method for grading the cognitive state of patients for the clinician. J Psychiatr Res. 1975;12(3):189-98.

30. FTLD Working Group of the ADC program. NACC Uniform Data Set (UDS)-FTLD Module Version 3.0. Seattle, WA: University of Washington; 2015.

31. Cummings JL. The neuropsychiatric inventory: assessing psychopathology in dementia patients. Neurology. 1997;48(5 Suppl 6): S10-6.

32. Jenkinson C, Fitzpatrick R, Peto V, Greenhall R, Hyman N. The Parkinson's disease Questionnaire (PDQ-39): development and validation of a Parkinson's disease summary index score. Age Ageing. 1997;26(5):353-7.

33. Goetz CG, Fahn S, Martinez-Martin P, et al. Movement Disorder Society-sponsored revision of the Unified Parkinson's Disease Rating Scale (MDS-UPDRS): process, format, and clinimetric testing plan. Mov Disord. 2007;22(1):41-7.

34. Hachinski VC, Iliff LD, Zilhka E, Cerebral blood flow in dementia. Arch Neurol. 1975;32(9):632-7.

35. Belleville S, Fouquet C, Duchesne S, Collins DL, Hudon C. Detecting early preclinical Alzheimer's disease via cognition, neuropsychiatry, and neuroimaging: qualitative review and recommendations for testing. J Alzheimers Dis. 2014;42:S375-82.

36. Stuss DT. The Ontario Brain Institute: completing the circle. Can J Neurol Sci. 2014;41(6):683-93.

37. Hallam BJ, Jacova C, Hsiung GY, et al. Early neuropsychological characteristics of progranulin mutation carriers. J Int Neuropsychol Soc. 2014;20(7):694-703.

38. de Frias CM, Dixon RA, Camicioli R. Neurocognitive speed and inconsistency in Parkinson's disease with and without incipient dementia: an 18-month prospective cohort study. J Int Neuropsychol Soc. 2012;18(4):764-72.

39. O'Bryant SE, Xiao G, Barber R, et al. A blood-based screening tool for Alzheimer's disease that spans serum and plasma: findings from TARC and ADNI. PLoS One. 2011;6(12):e28092.

40. Agarwal R, Tripathi CB. Diagnostic utility of CSF Tau and Abeta(42) in dementia: a meta-analysis. Int $\mathbf{J}$ Alzheimers Dis. 2011;2011:503293.

41. Pekeles H, Qureshi HY, et al. Development and validation of a salivary tau biomarker in Alzheimer disease. Alzheimers Dement. 2019;11:53-60.

42. Vellas B, Hampel H, Rougé-Bugat ME, et al. Alzheimer's disease therapeutic trials: EU/US Task Force report on recruitment, retention, and methodology. J Nutr Health Aging. 2012; 16(4):339-45.

43. Duchesne $\mathrm{S}$, Valdivia $\mathrm{F}$, Robitaille $\mathrm{N}$ et al. Manual segmentation qualification platform for the EADC-ADNI harmonized protocol 
for hippocampal segmentation project. Alzheimers Dement. 2015;11(2):161-74.

44. Anandh KR, Sujatha CM, Ramakrishnan S. A method to differentiate mild cognitive impairment and Alzheimer in MR images using Eigen value descriptors. J Med Syst. 2016;40(1):25.

45. Wardlaw JM, Smith EE, Biessels GJ, et al. Neuroimaging standards for research into small vessel disease and its contribution to ageing and neurodegeneration. Lancet Neurol. 2013;12(8):822-38.

46. Fazekas F, Kleinert R, Offenbacher H, et al. Pathologic correlates of incidental MRI white matter signal hyperintensities. Neurology. 1993;43(9):1683-9.

47. Hardy J, Orr H. The genetics of neurodegenerative diseases. J Neurochem. 2006;97(6):1690-9.

48. Caracciolo B, Xu W, Collins S, Fratiglioni L. Cognitive decline, dietary factors and gut-brain interactions. Mech Ageing Dev. 2015;136-137:59-69.

49. Hill JM, Clement C, Pogue AI, Bhattacharjee S, Zhao Y, Lukiw WJ. Pathogenic microbes, the microbiome, and Alzheimer's disease (AD). Front Aging Neurosci. 2014;6:127.

50. Bhattacharjee S, Lukiw WJ. Alzheimer's disease and the microbiome. Front Cell Neurosci. 2013;7:153.
51. Mayer EA, Knight R, Mazmanian SK, Cryan JF, Tillisch K. Gut microbes and the brain: paradigm shift in neuroscience. J Neurosci. 2014;34(46):15490-6.

52. Zapata HJ, Quagliarello VJ. The microbiota and microbiome in aging: potential implications in health and age-related diseases. $\mathrm{J}$ Am Geriatr Soc. 2015;63(4):776-81.

53. Hill JM, Bhattacharjee S, Pogue AI, Lukiw WJ. The gastrointestinal tract microbiome and potential link to Alzheimer's disease. Front Neurol. 2014;5:43.

54. Tremlett H, Bauer KC, Appel-Cresswell S, Finlay BB, Waubant E. The gut microbiome in human neurological disease: a review. Ann Neurol. 2017;81(3):369-82.

55. Mather KA, Kwok JB, Armstrong N, Sachdev PS. The role of epigenetics in cognitive ageing. Int $\mathrm{J}$ Geriatr Psychiatry. 2014;29(11):1162-71.

56. Akbarian S, Beeri MS, Haroutunian V. Epigenetic determinants of healthy and diseased brain aging and cognition. JAMA Neurol. 2013;70(6):711-8.

57. Ashish N, Bhatt P, Toga AW. Global data sharing in Alzheimer disease research. Alzheimer Dis Assoc Disord. 2016;30(2):160-8. 